\title{
Geranylgeranyl diphosphate synthase inhibitor and proteasome inhibitor combination therapy in multiple myeloma
}

Staci L. Haney', Michelle L. Varney', Jacob T. Williams' ${ }^{1}$, Lynette M. Smith², Geoffrey Talmon ${ }^{3}$ and

Sarah A. Holstein ${ }^{1 *}$ (1)

\begin{abstract}
Background: Multiple myeloma (MM) remains an incurable malignancy, despite the advent of therapies such as proteosome inhibitors (PIs) that disrupt protein homeostasis and induce ER stress. We have pursued inhibition of geranylgeranyl diphosphate synthase (GGDPS) as a novel mechanism by which to target protein homeostasis in MM cells. GGDPS inhibitors (GGSI) disrupt Rab geranylgeranylation, which in turn results in perturbation of Rab-mediated protein trafficking, leading to accumulation of intracellular monoclonal protein, induction of ER stress and apoptosis. Our lead GGSI, RAM2061, has demonstrated favorable pharmacokinetic properties and in vivo efficacy. Here we sought to evaluate if combination therapy with GGSI and PI would result in enhanced disruption of the unfolded protein response (UPR) and increase anti-MM efficacy.
\end{abstract}

Methods: MTT assays were conducted to evaluate the cytotoxic effects of combining RAM2061 with bortezomib in human MM cells. The effects of RAM2061 and/or PI (bortezomib or carfilzomib) on markers of UPR and apoptosis were evaluated by a combination of immunoblot (ATF4, IRE1, p-elF2a, cleaved caspases and PARP), RT-PCR (ATF4, ATF6, CHOP, PERK, IRE1) and flow cytometry (Annexin-V). Induction of immunogenic cell death (ICD) was assessed by immunoblot (HMGB1 release) and flow cytometry (calreticulin translocation). Cell assays were performed using both concurrent and sequential incubation with PIs. To evaluate the in vivo activity of GGSI/PI, a flank xenograft using MM.1S cells was performed.

Results: Isobologram analysis of cytotoxicity data revealed that sequential treatment of bortezomib with RAM2061 has a synergistic effect in MM cells, while concurrent treatment was primarily additive or mildly antagonistic. The effect of PIs on augmenting RAM2061-induced upregulation of UPR and apoptotic markers was dependent on timing of the PI exposure. Combination treatment with RAM2061 and bortezomib enhanced activation of ICD pathway markers. Lastly, combination treatment slowed MM tumor growth and lengthened survival in a MM xenograft model without evidence of off-target toxicity.

Conclusion: We demonstrate that GGSI/PI treatment can potentiate activation of the UPR and apoptotic pathway, as well as induce upregulation of markers associated with the ICD pathway. Collectively, these findings lay the groundwork for future clinical studies evaluating combination GGSI and PI therapy in patients with MM.

*Correspondence: sarah.holstein@unmc.edu

${ }^{1}$ Division of Oncology and Hematology, Department of Internal Medicine, University of Nebraska Medical Center, Omaha, NE 68198, USA

Full list of author information is available at the end of the article

\section{Background}

Multiple myeloma (MM) is a blood cancer caused by the malignant proliferation of clonal plasma cells in the bone marrow. MM accounts for $10 \%$ of all hematological cancers and approximately 35,000 new cases will be 
diagnosed in 2021. MM cells produce and secrete large quantities of monoclonal proteins (MP), which can be detected in the peripheral blood. To alleviate endoplasmic reticulum (ER) stress from the continuous manufacturing of MP, MM cells express near maximal levels of unfolded protein response (UPR) associated proteins and therefore are particularly sensitive to activation of the pro-apoptotic arm of the UPR [1,2]. Disruption of the machinery regulating protein homeostasis and the resulting induction of ER stress-associated apoptosis serves as a targeted therapeutic strategy against MM [3-5]. Current front-line and relapsed/refractory treatments for MM include proteasome inhibitors (PIs), which work by blocking the proteasome from recycling misfolded proteins, leading to ER stress and apoptosis [6-8]. The FDA approved the first-in-class PI, bortezomib, in 2003 followed by the second-generation PI, carfilzomib, in 2012. While PIs have proven very beneficial for the treatment of MM, development of relapsed/refractory MM remains a significant clinical challenge [9-11] and necessitates the need for novel therapies.

As an alternative strategy by which to target protein homeostasis in MM, we have focused on the development of geranylgeranyl diphosphate synthase (GGDPS) inhibitors (GGSIs) [12-21]. In the isoprenoid biosynthetic pathway (IBP), GGDPS catalyzes the synthesis of the isoprenoid donor (GGPP) which in turn is covalently linked to the target Rab proteins by the enzyme geranylgeranyl transferase (GGTase) II. Rabs regulate nearly all intracellular trafficking events and require geranylgeranylation in order to be properly localized and functional [22]. We have shown that agents that impair Rab geranylgeranylation induce MM cell death by disrupting the trafficking of MP, resulting in induction of the UPR and apoptosis [23-25]. We have previously reported preclinical studies with our lead GGSI, RAM2061, which demonstrated the agent's metabolic stability, prolonged half-life, systemic distribution, in vivo disruption of geranylgeranylation and anti-tumor efficacy in a mouse MM xenograft model [24].

We hypothesized that the combination of GGSI and PI therapy would result in enhanced activation of the UPR and increase anti-MM efficacy. Here we demonstrate that combination treatment with RAM2061 and PI (bortezomib or carfilzomib) enhances UPR activation and MM cell death in vitro when compared to single drug treatment, and that these effects are dependent on the timing of drug exposure. In addition, combination therapy induces activation of the immunogenic cell death pathway (ICD). Furthermore, combination GGSI and bortezomib treatment significantly slowed in vivo tumor growth and lengthened survival relative to single drug treatments in a mouse MM xenograft model.
Collectively, these studies are the first to demonstrate the feasibility and therapeutic benefit of combining GGSI and PIs and lay the foundation for future clinical studies.

\section{Methods \\ Chemicals}

Dr. David Wiemer at the University of Iowa kindly provided RAM2061 [18]. Compound purity was determined as $\geq 95 \%$ by high-performance liquid chromatography and verified by nuclear magnetic resonance [18]. Bortezomib was purchased from Millipore sigma (Burlington, MA, USA) and carfilzomib was purchased from Amgen (Thousand Oaks, CA, USA).

\section{Cell culture}

MM.1S and RPMI-8226 cells were obtained from American Type Culture Collection (Manassas, VA, USA). JJN3 cells were obtained from DSMZ-German Collection of Microorganisms and Cell Cultures. ALMC-2 cells [26] were obtained from Dr. Diane Jelinek, Mayo Clinic (Rochester, MN). Cells were grown in media supplemented with $10 \%$ heat-inactivated fetal bovine serum (20\% for JJN3), glutamine, and penicillin-streptomycin at $37{ }^{\circ} \mathrm{C}$ and $5 \% \mathrm{CO}_{2}$. Mycoplasma testing was performed using MycoAlert mycoplasma detection kit (Lonza, Rockland, ME, USA).

\section{MTT}

Cells were plated $\left(3.5 \times 10^{4}\right.$ cells $/ 150 \mu \mathrm{L}$ per well $)$ in 96-well plates and incubated in the presence or absence of drug. The MTT assay was performed as previously described [27]. Data were normalized so that the absorbance acquired from cells treated with solvent only was defined as having an MTT activity of $100 \%$.

\section{ELISA}

A human lambda ELISA kit (Bethyl Laboratories, Montgomery, TX, USA) was used to quantify intracellular monoclonal protein levels from the cell culture studies (ALMC-2, RPMI-8226 and MM.1S) as well as the levels of human lambda light chain in the blood samples from the xenograft study. Intracellular kappa light chain levels were detected in JJN3 cells using a human kappa ELISA kit (Bethyl Laboratories).

\section{Detection of apoptosis by flow cytometry}

Cells were incubated for $48 \mathrm{~h}$ in the presence of RAM2061. PI (bortezomib or carfilzomib) was added either concurrently or sequentially (24 h after RAM2061). Cells were washed with PBS and stained with APC-conjugated Annexin $\mathrm{V}$ antibody and propidium iodide per the manufacturer's instruction (eBioscience, San Diego, CA, USA). Ten-thousand cell events were recorded using 
a BD LSRII flow cytometer. FlowJo software was used for all data analysis. We define early apoptotic cells as being Annexin $\mathrm{V}+/$ Propidium iodide - and late apoptotic as Annexin $\mathrm{V}+/$ Propidium iodide + .

\section{Detection of cell surface calreticulin}

Cells were incubated for $48 \mathrm{~h}$ in the presence of RAM2061. PI (bortezomib or carfilzomib) was added either concurrently or sequentially (24 h after RAM2061). Cells were washed with PBS and incubated with calreticulin polyclonal antibody (1:200, Invitrogen PA3-900), followed by secondary anti-rabbit Alexafluor594. Tenthousand cell events were recorded using a BD LSRII flow cytometer. FlowJo software was used for all data analysis.

\section{Immunoblot}

Cells were incubated for the specified amount of time in the presence or absence of RAM2061, bortezomib or carfilzomib. Cells were washed with phosphate-buffered saline (PBS) and lysed in RIPA buffer (1\% sodium deoxycholate, $0.15 \mathrm{M} \mathrm{NaCl}, 0.1 \%$ SDS, $1 \%(\mathrm{v} / \mathrm{v})$ Triton X-100, $0.05 \mathrm{M}$ Tris $\mathrm{HCl}, \mathrm{pH}$ 7.4) supplemented with protease and phosphatase inhibitors. Protein concentration was determined using the BCA method. Protein $(15 \mu \mathrm{g})$ was run on an SDS-PAGE gel and transferred to a polyvinylidene difluoride membrane. Blots were incubated overnight in primary antibody and for $1 \mathrm{~h}$ in secondary antibody. Protein was visualized using a chemiluminescence detection kit and a Bio-Rad Chemidoc MP imaging system. Antibodies used for these assays can be found in the Additional file 1: Table S1.

\section{Quantitative real-time polymerase chain reaction}

Cells were incubated for the specified amount of time in the presence or absence of RAM2061, bortezomib or carfilzomib. RNA was isolated using the E.Z.N.A HP total RNA kit from Omega Biotek (Norcross, GA, USA). RNA $(1 \mu \mathrm{g})$ was reverse-transcribed to cDNA using the i-Script cDNA synthesis kit (Bio-Rad, Hercules, CA) and mixed with gene-specific primers and i-Taq Sybr green super mix (Bio-Rad) according to manufacturer's instruction. qRT-PCR reactions were performed in triplicate using a Bio-Rad CFX96 instrument. Data analysis was performed using the Bio-Rad CFX manager 3.1 software. Gene expression was normalized to that of $\beta$-actin. Primer sequences used in these studies can be found in Additional file 1: Table S2.

\section{Myeloma xenografts}

Female NOD/SCID mice (Charles River) between the ages of 6-8 weeks were housed in the University of Nebraska Medical Center (UNMC) animal facility at a temperature of $23-25{ }^{\circ} \mathrm{C}$, relative humidity of $50-70 \%$ and 12/12-h light/dark cycles. The UNMC IACUC (protocol number 16-132-11-FC) approved all animal studies. NOD-SCID mice were subcutaneously inoculated in the flank with MM.1S cells (10 million cells in $0.1 \mathrm{~mL}$ of sterile saline mixed 1:1 with Matrigel). When tumors became palpable (approximately 2 weeks), mice were divided into control and treatment groups to provide an equal distribution of tumor size for each group at the start of treatment $(n=8)$. Our pre-established practice is to exclude mice that do not have palpable tumors by the start of treatment. Thus, one mouse from both the control and RAM2061 group were excluded due to lack of tumor engraftment. Controls were administered 100 $\mu \mathrm{L}$ PBS $2 \times / \mathrm{wk}$ (IV). Bortezomib was administered subcutaneously at a dose of $0.3 \mathrm{mg} / \mathrm{kg} 2 \times / \mathrm{wk}$. RAM2061 was administered IV at a dose of $0.08 \mathrm{mg} / \mathrm{kg} 2 \times / \mathrm{wk}$. All mice began treatment on day 14 post MM cell inoculation. Tumor volume was recorded three times per week using a caliper. Investigators were not blinded during the course of this experiment. The following equation was used to calculate tumor volume: $4 \pi / 3 \times(\text { width } / 2)^{\wedge} 2 \times(1$ ength/2).

\section{Blood analysis}

Blood samples were obtained postmortem from the heart and analyzed using an Abaxis Vetscan2 instrument and the preventive profile plus rotor (Allied Analytic, Tampa, FL).

\section{Histology}

Mouse organs were fixed in formalin for 24-96 h, stored in $70 \%$ ethanol, embedded in paraffin, sectioned, and stained (H\&E or with anti-cleaved caspase 3 antibody) using standard methods at the UNMC Tissue Science Facility. To quantify the cleaved caspase 3 data, stained cells from five fields of view/sample were manually counted (ImageJ software) and averages were calculated for each sample.

\section{Statistics}

T-tests (two-tailed) were used to calculate statistical significance between control and treated groups. Linear mixed models (LMM) were used to look at changes in tumor burden over time. Tumor burden was modeled on the $\log _{10}$ scale in order to meet model assumptions. The model included fixed effects for group, day and the group $\mathrm{x}$ day interaction and day is modeled as a continuous variable. A random slope and intercept were fit for each mouse. Distributional assumptions of the LMM were assessed using residual plots and the linearity and equal variance assumptions were found to be met when data was analyzed on the $\log _{10}$ scale. Kaplan-Meier 
Table 1 Summary of combination indices from MTT cytotoxicity studies for sequential bortezomib and RAM2061 treatment

\begin{tabular}{lllll}
\hline Fa & ALMC-2 & JJN3 & MM.1S & RPMI \\
\hline 0.3 & 0.51 & 0.94 & 0.97 & 0.14 \\
0.5 & 0.34 & 0.94 & 0.87 & 0.06 \\
0.75 & 0.2 & 0.96 & 0.77 & 0.03 \\
\hline
\end{tabular}

Cells were incubated with varying concentrations of RAM2061 and/or bortezomib. RAM2061 was added at the beginning of the 48-h incubation period. Studies were performed in which bortezomib was added $24 \mathrm{~h}$ after RAM2061 (sequential). Isobologram analysis was performed, and combination indices were determined. Combination indices $<1$ are considered synergistic in nature

Table 2 Summary of combination indices from MTT cytotoxicity studies for concurrent bortezomib and RAM2061 treatment

\begin{tabular}{lllll}
\hline Fa & ALMC-2 & JJN3 & MM.1S & RPMI \\
\hline 0.3 & 1.81 & 1.22 & 1.45 & 1.36 \\
0.5 & 1.95 & 1.25 & 1.5 & 1.37 \\
0.75 & 2.2 & 1.35 & 1.58 & 1.38 \\
\hline
\end{tabular}

Cells were incubated with varying concentrations of RAM2061 and/or bortezomib. RAM2061 and bortezomib were added at the beginning of the 48-h incubation period (concurrent). Isobologram analysis was performed, and combination indices were determined. Combination indices $>1$ are considered antagonistic in nature

method was used to estimate overall survival distributions by treatment group. Overall survival was defined as time from start of treatment to death. A log rank test with was used to calculate statistical significance of the Kaplan-Meier survival curve and the Holm-Sidak method was applied to adjust for multiple comparisons. GraphPad Prism version 7.04 (San Diego, CA, USA) and SAS software version 9.4 (Cary, NC, USA) were used for data analysis. With $\mathrm{n}=8$ mice per group we achieve $80 \%$ power to detect a mean difference in tumor burden of $200 \mathrm{~mm}^{3}$ with a $\mathrm{SD}=100 \mathrm{~mm}^{3}$ and alpha $=0.008$ (Bonferroni adjusted for 6 pairwise comparisons) using a twosided two-sample equal-variance t-test.

\section{Results}

\section{Effects of combination therapy on MM cell death}

As an initial evaluation of the cytotoxic effects of combining the GGSI RAM2061 with the PI bortezomib in MM cells, MTT assays were conducted in four human MM cell lines (MM.1S, RPMI-8226, JJN3, and ALMC2). RAM2061 was added at the beginning of the 48-h incubation period while bortezomib was added at either the same time (concurrent) or after $24 \mathrm{~h}$ (sequential). Isobologram analysis of the MTT data was performed and combination indices (CI) were determined (Tables 1 and 2). These studies revealed that sequential treatment of bortezomib with RAM2061 resulted in a primarily synergistic interaction $(\mathrm{CI}<1)$ while concurrent treatment was primarily additive $(\mathrm{CI}=1)$ to antagonistic $(\mathrm{CI}>1)$ (Additional file 1: Fig. S1). Additional MTT assays were performed using the PI carfilzomib. We also observed primary synergistic $(\mathrm{Cl}<1)$ to additive $(\mathrm{CI}=1)$ interactions following sequential treatment of carfilzomib with RAM2061, and a primarily additive $(\mathrm{CI}=1)$ to antagonistic $(\mathrm{CI}>1)$ interaction when using concurrent carfilzomib and RAM2061 (Additional file 1: Fig. S2, Table S3).

To explore further the impact of timing of PI administration on induction of cell death with RAM2061, we assessed induction of apoptosis by flow cytometry using Annexin $\mathrm{V}$ and propidium iodide staining. Relative to single treatment controls, both concurrent and sequential treatment of bortezomib with RAM2061 lead to an increase in the percent of apoptotic cells (Fig. 1). Conversely, we did not observe a similar increase in apoptotic cells in cell lines treated with RAM2061 in combination with another PI, carfilzomib (Additional file 1: Fig. S3). We also examined induction of apoptosis via immunoblot analysis of cleaved PARP, caspases 3, 8, and 9. MM cells were incubated in the presence or absence of RAM2061 (50 or $100 \mathrm{nM}$ ) for $48 \mathrm{~h}$. PI was added concurrent with RAM2061 or either $6 \mathrm{~h}$ (denoted as $*$ ) or $24 \mathrm{~h}$ (denoted as *) prior to cell harvest. We observed increased PARP and caspase cleavage in MM cells treated with RAM2061 and 6-h sequential treatment of either bortezomib or carfilzomib when compared to single drug treatment (Fig. 2a). Likewise, MM cells treated with RAM2061 and 24-h sequential bortezomib or carfilzomib displayed enhanced apoptotic markers relative to single treatment controls (Fig. 2b). Conversely, concurrent treatment with PI resulted in more rapid cell death and a consequent decrease in protein levels was observed (Additional file 1: Fig. S4).

\section{Comparison of UPR induction with concurrent vs sequential PI therapy in combination with RAM2061} Next, we examined markers of the UPR (ATF4, IRE1 and phosphorylated-eIF2 $\alpha$ (p-eIF2 $\alpha)$ ) using the same sequential and concurrent PI treatment as previously described. These studies revealed that administration of either bortezomib or carfilzomib during the final $6 \mathrm{~h}$ or final $24 \mathrm{~h}$ of incubation enhanced the RAM2061-induced upregulation of UPR markers in a cell line-specific manner (Fig. 3a and b). Concurrent 48-h treatment with PI led to rapid apoptosis and a subsequent decrease in UPR markers, likely due to the rapid effects of PI on the UPR pathway (Additional file 1: Fig. S5). qRT-PCR analysis of UPR markers revealed a partial and cell line-dependent increase in ATF4, AT6, CHOP, IRE1 and PERK expression levels following 6- and 24-h incubation of PI with 

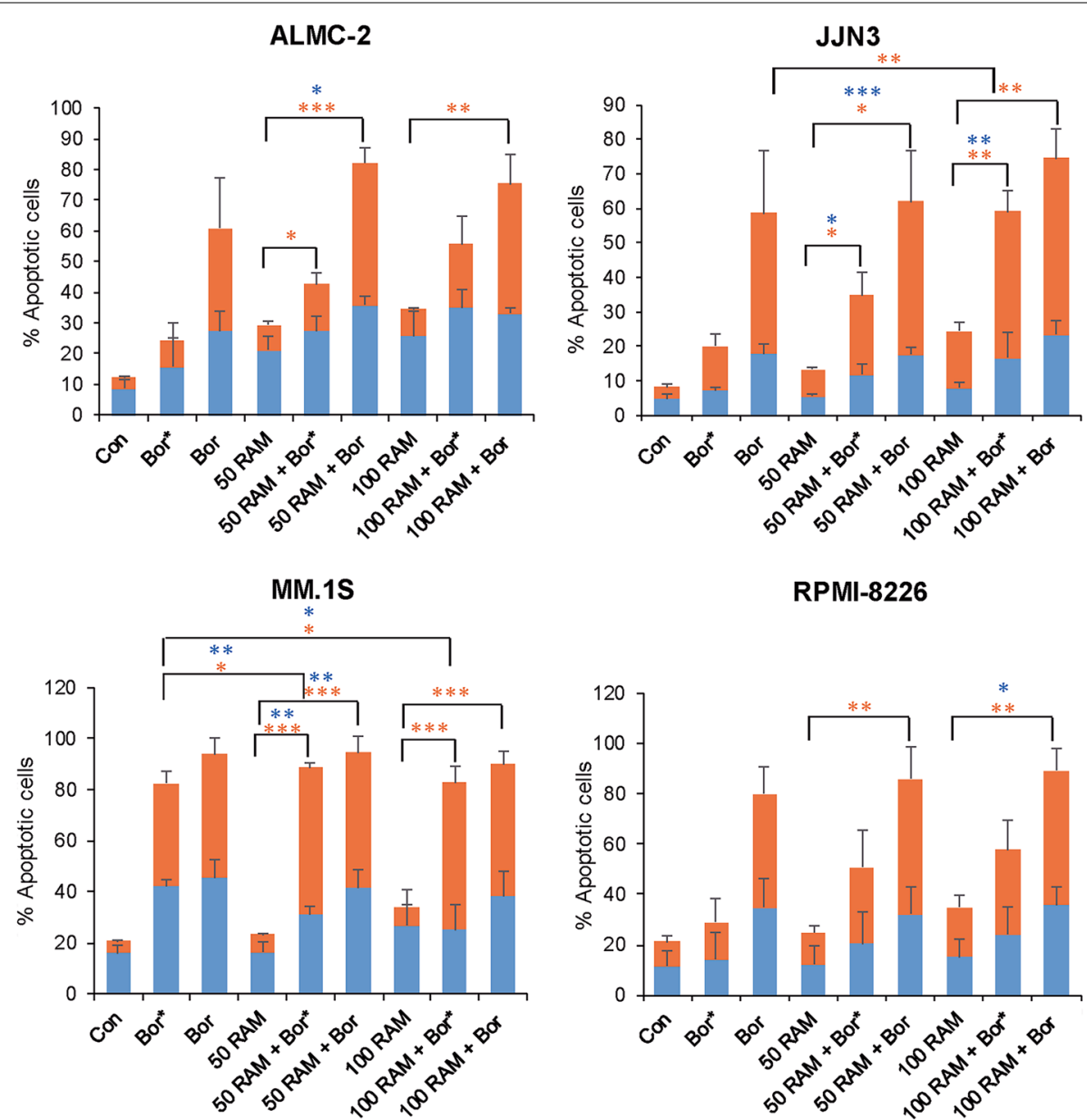

RPMI-8226

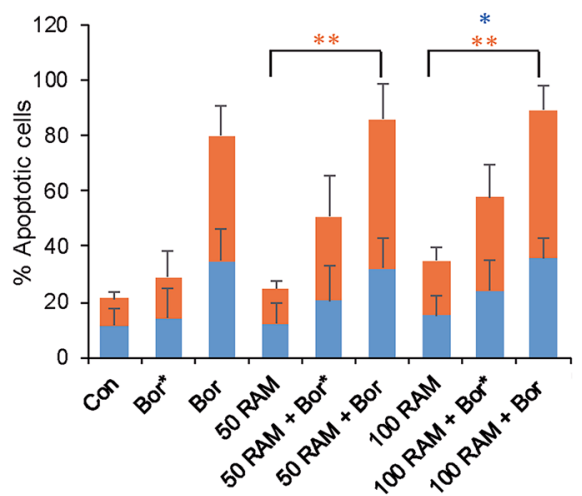

Early apo Late apo

Fig. 1 Effects of sequential and concurrent bortezomib therapy in combination with RAM2061 on induction of apoptosis. MM cells were incubated with RAM2061 (50 or $100 \mathrm{nM}$ ) for 48 h. Bortezomib was added either concurrently or after $24 \mathrm{~h}$ (sequential; denoted by *) (Bor: 3 nM (MM.1S), 5 nM (JJN3 and RPMI-8226), 15 nM (ALMC-2)). Cells were stained with fluorescently conjugated Annexin V and propidium iodide (PI) and analyzed by flow cytometry. Data are expressed as the average percentage of Annexin V+/PI- (early apoptotic) and Annexin V+/PI+ (late apoptotic) ( $n=3$, data are displayed as mean \pm stdev, ${ }^{*}$ denotes $p<0.05 .{ }^{* *}$ denotes $p<0.01 .{ }^{* *}$ denotes $p<0.001$ per t-test)

RAM2061 relative to single treatment controls (Additional file 1: Fig. S6 and S7). Neither bortezomib nor carfilzomib enhanced RAM2061-mediated intracellular light chain retention as measured by ELISA (Additional file 1: Fig. S8).

\section{Combination treatment with RAM2061 and PI leads to induction of the ICD pathway}

Next, we sought to determine if GGSI treatment, either alone or in combination with a PI, could induce the immunogenic cell death (ICD) pathway. This pathway is characterized by cell-surface translocation of calreticulin, release of damage-associated molecular pattern (DAMP) molecules (including HMGB1 (high mobility group protein B1)) and stimulation of type I interferon (IFN) responses. To measure HMBGI release, we performed immunoblot analysis on media collected from MM cells incubated with RAM2061 and PI. Both 24- and 48-h PI treatment in the presence of RAM2061 enhanced the release of HMGB1 into the media (Fig. 4a). Bortezomib induced cell-surface translocation of calreticulin following both 24- and 48-h incubation in all four cell lines, while carfilzomib and RAM2061 had little effect on cell surface levels of calreticulin when used as single agents (Fig. 4b, c and Additional file 1: Fig. S9). Both concurrent and sequential incubation of bortezomib in the presence of RAM2061 enhanced cell surface translocation of calreticulin relative to single drug treatments (Fig. 4b, c). 


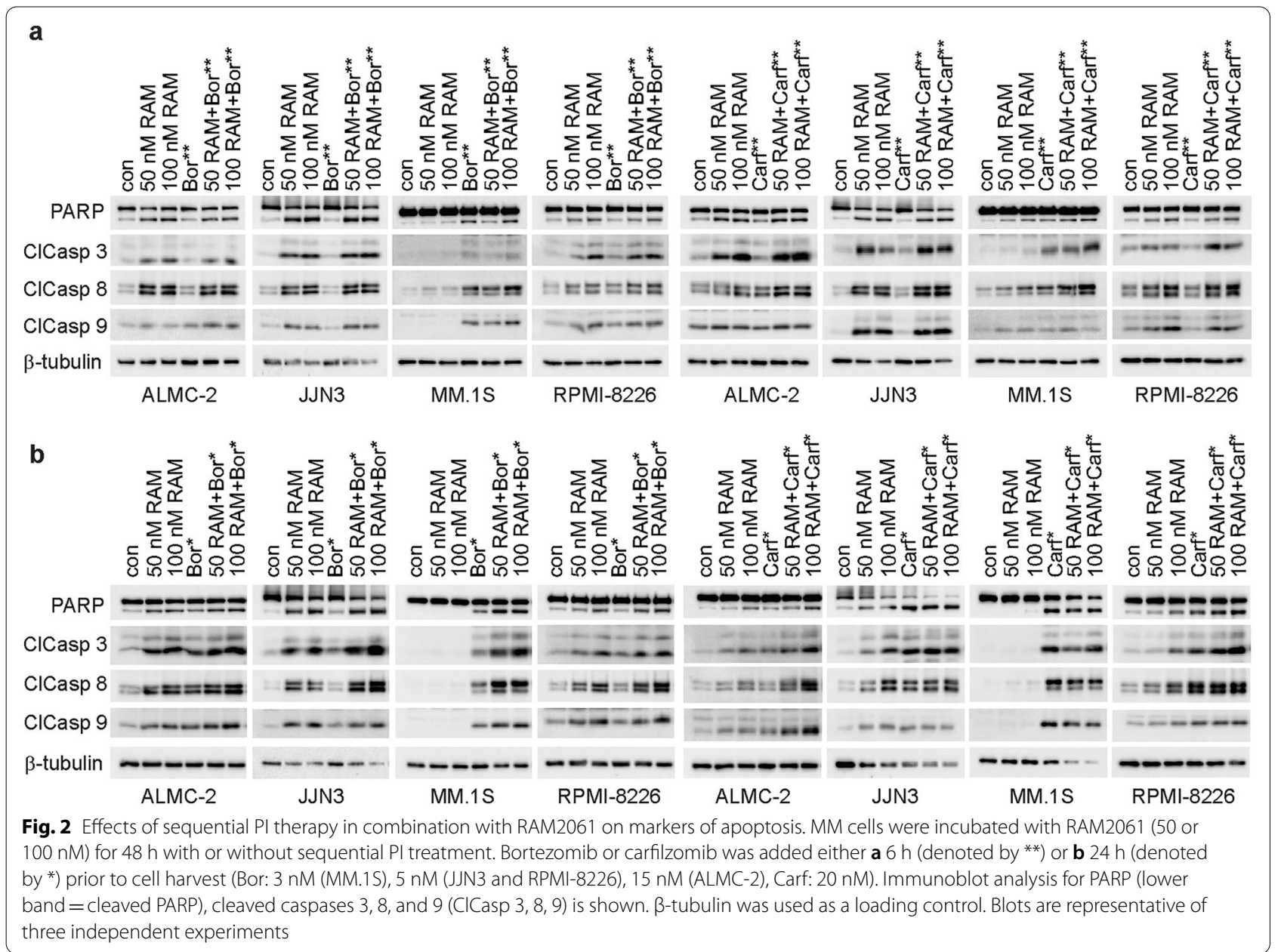

\section{Effects of RAM2061 and/or bortezomib treatment in MM mouse xenografts}

Using a MM flank xenograft model, we examined if combination treatment of bortezomib with RAM2061 would be efficacious in slowing tumor growth in vivo. NODSCID mice were inoculated with MM.1S cells in the flank and treated with PBS (control), bortezomib, RAM2061, or a combination of RAM2061/bortezomib. Tumor growth over time was significantly decreased in the RAM2061 ( $\mathrm{p}=0.0009$ ) and in the RAM2061/bortezomib $(\mathrm{p}=0.0002)$ treatment groups compared to control animals (Fig. 5a). In addition, survival, or time to sacrifice, was significantly prolonged in the RAM2061/bortezomib cohort $(p=0.003)$, but not the RAM2061 group $(p=0.09$, Fig. 5b). Concordant with tumor volume, mean plasma human lambda light chain levels were decreased in the RAM2061 and to a larger degree in the RAM2061/ bortezomib treatment groups when compared to the control mice ( $\mathrm{p}<0.05$, Fig. $5 \mathrm{c}$ ). Consistent with our previous in vivo studies, the primary toxicity associated with RAM2061 treatment was hepatic transaminase elevation
(Additional file 1: Table S4). Histological examination of livers revealed mild and variable lobular unrest (defined as variable nuclear enlargement with a rare binucleated hepatocyte) around the central vein (zone 3) likely related to mild regenerative changes in bortezomib treated mice (Additional file 1: Fig. S10). Livers of RAM2061-treated mice displayed prominent lobular unrest around zone 3 with rare apoptotic hepatocytes and occasional lipofuscin-laden macrophages which signify hepatocyte dropout. Livers of mice treated with both drugs showed the same prominent lobular in zone 3 with adjacent ballooning degeneration in zone 2 hepatocytes. Spleen and kidney morphology were normal across all groups (Additional file 1: Fig. S10). No additional toxicity was observed when bortezomib was combined with RAM2061 based on animal weight, renal or hepatic parameters (Additional file 1: Fig. S11 and Table S4). We observed a trend toward greater cleaved caspase 3 expression in tumors isolated from mice treated with RAM2061 relative to controls (Additional file 1: Fig. S12). 


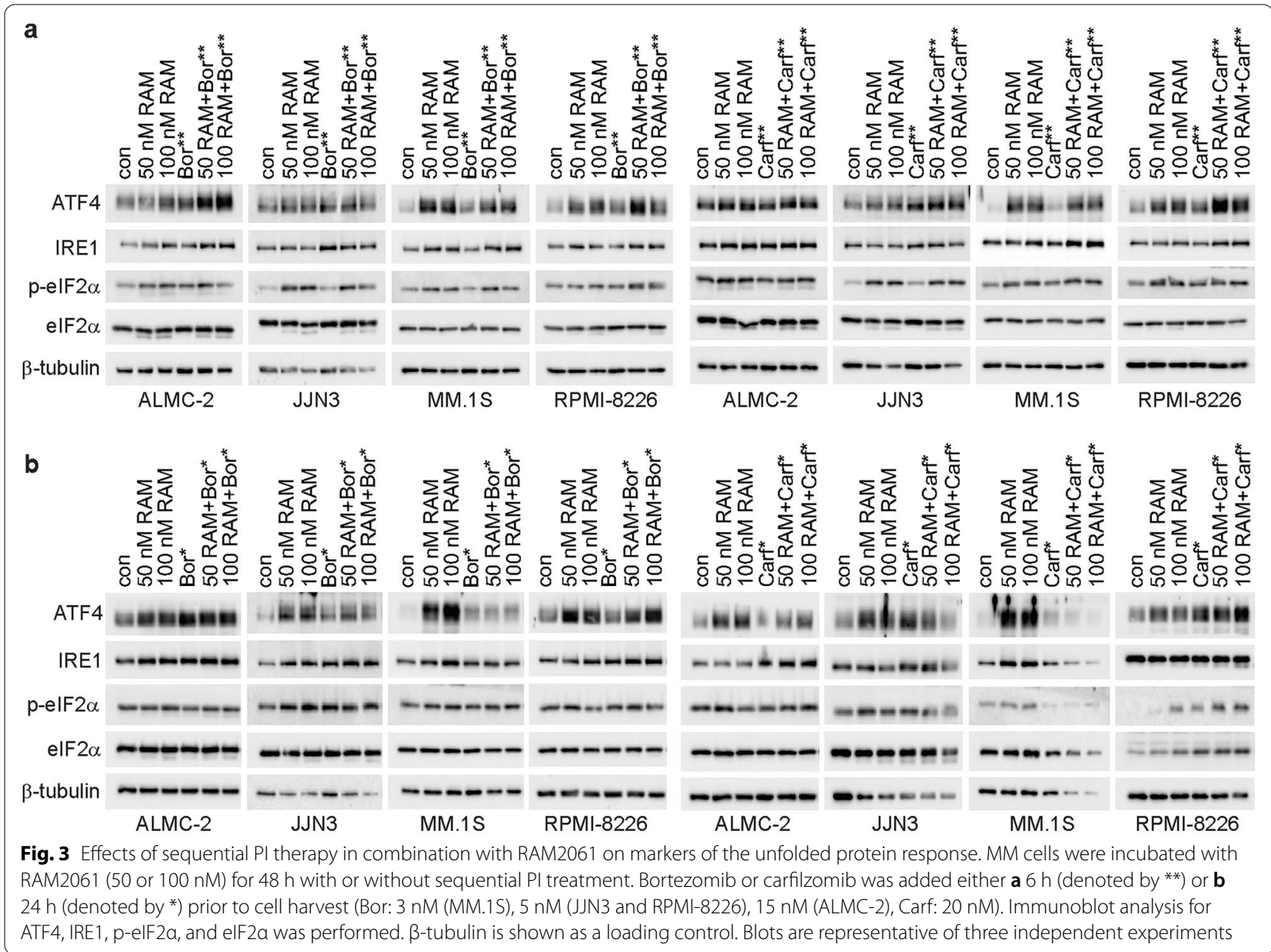

\section{Discussion}

RAM2061 is a homoneryl triazole bisphosphate that functions as a potent GGDPS inhibitor with an $\mathrm{IC}_{50}$ of $86 \mathrm{nM}$ and lowest effective concentration in MM cells of $25 \mathrm{nM}$ [18]. Our preclinical studies demonstrate that RAM2061 displays a prolonged terminal half-life of $29.2 \mathrm{~h}$, metabolic stability, in vivo on target effects, and systemic distribution including favorable bone marrow exposure [24]. In addition, single agent activity was observed in a xenograft model using human MM.1S cells. Notably, this is the first study to show that RAM2061 and bortezomib have synergistic cytotoxic activity in MM cells. Further investigation revealed that bortezomib and carfilzomib are able to potentiate induction of markers of the UPR and ICD pathways in the presence of RAM2061. In addition, we present the first in vivo investigation showing the anti-tumor effects of combination GGSI/PI inhibitor therapy in a xenograft tumor model.

The proteasome is tasked with degrading the majority of cellular proteins and thus plays a pivotal role in maintaining protein homeostasis. PIs induce MM cell death via various well-documented mechanisms, including the inhibition of several pro-survival factors, such cytokines, adhesion molecules, angiogenesis, and NF- $\mathrm{kB}$ $[6-8,28]$. In addition, PI-mediated accumulation of misfolded protein and induction of the UPR in MM cells has been described by several accounts in the literature. One study showed that bortezomib induced the accumulation of misfolded ER-processed proteins, which led to activation of the pro-apoptotic arm of the UPR, and that MM cells sensitivity to bortezomib directly correlates with the amount of MP retained in the cell [1]. In another study, a specific inhibitor of the proteasome $\beta 2$ subunit activated the UPR and induced cytotoxicity in bortezomib-resistant MM cells when combined with either bortezomib or carfilzomib [29]. Dual inhibition of the $\beta 2$ and $\beta 5$ subunits using a PI termed syringolog-1 resulted in increased expression of CHOP, ATF3, and $\mathrm{XBP} 1$, as well as induction of apoptosis in both bortezomib resistant and sensitive cell lines [30]. Likewise, we observed upregulation of several UPR mediators upon bortezomib or carfilzomib treatment, including ATF4, 


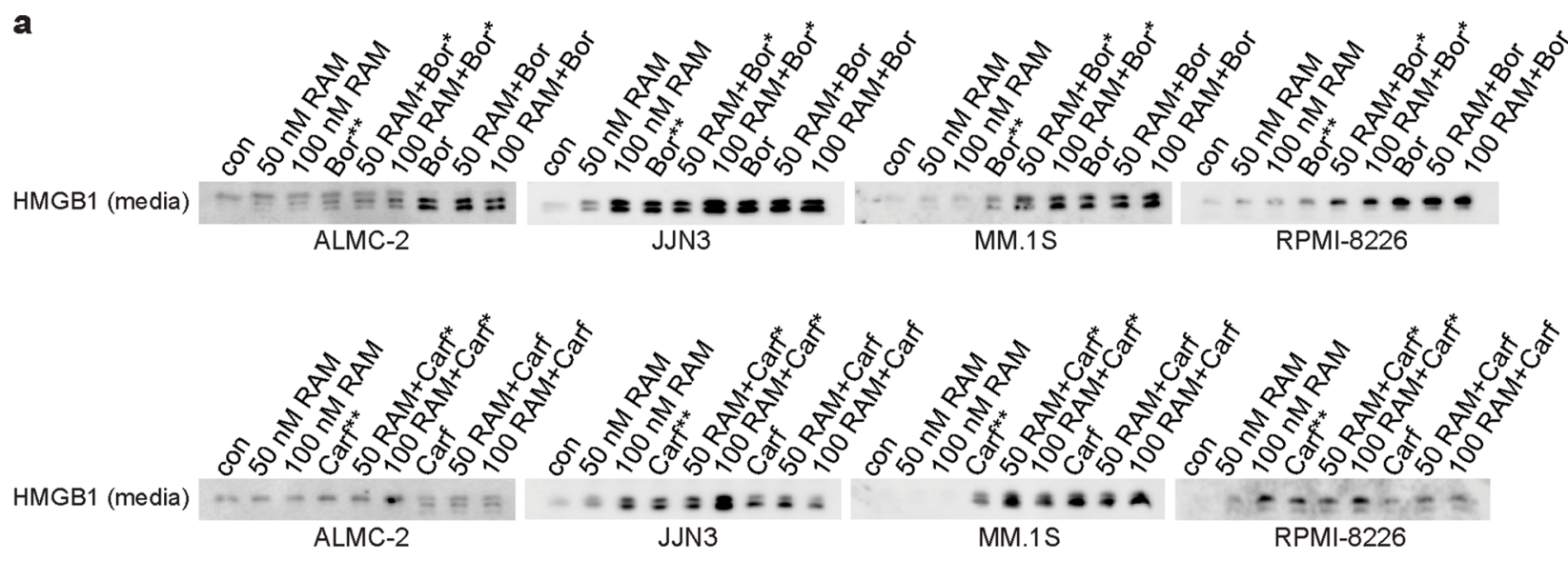

b

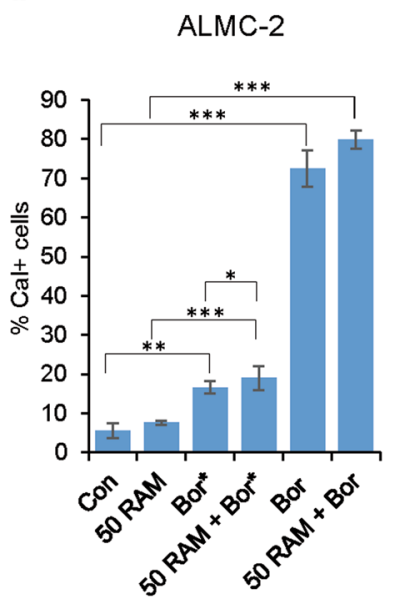

MM.1S

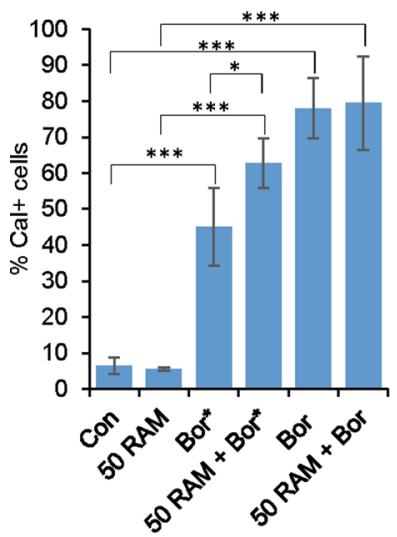

JJN3

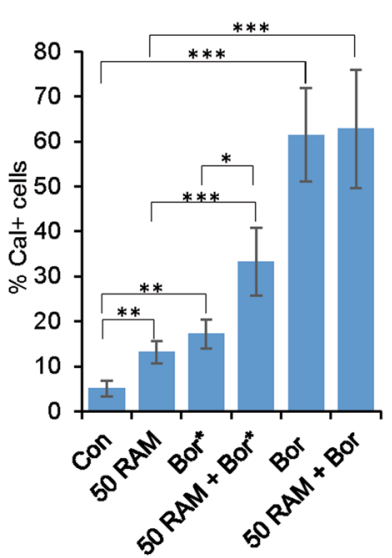

RPMI-8226

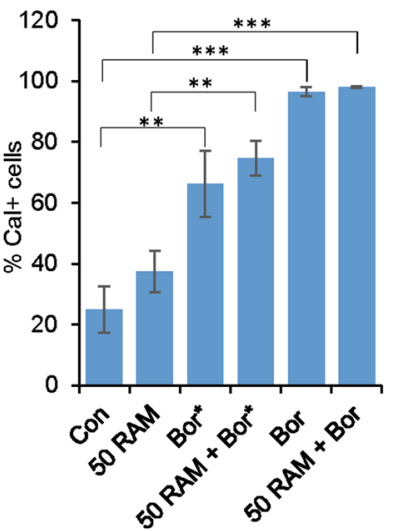

C
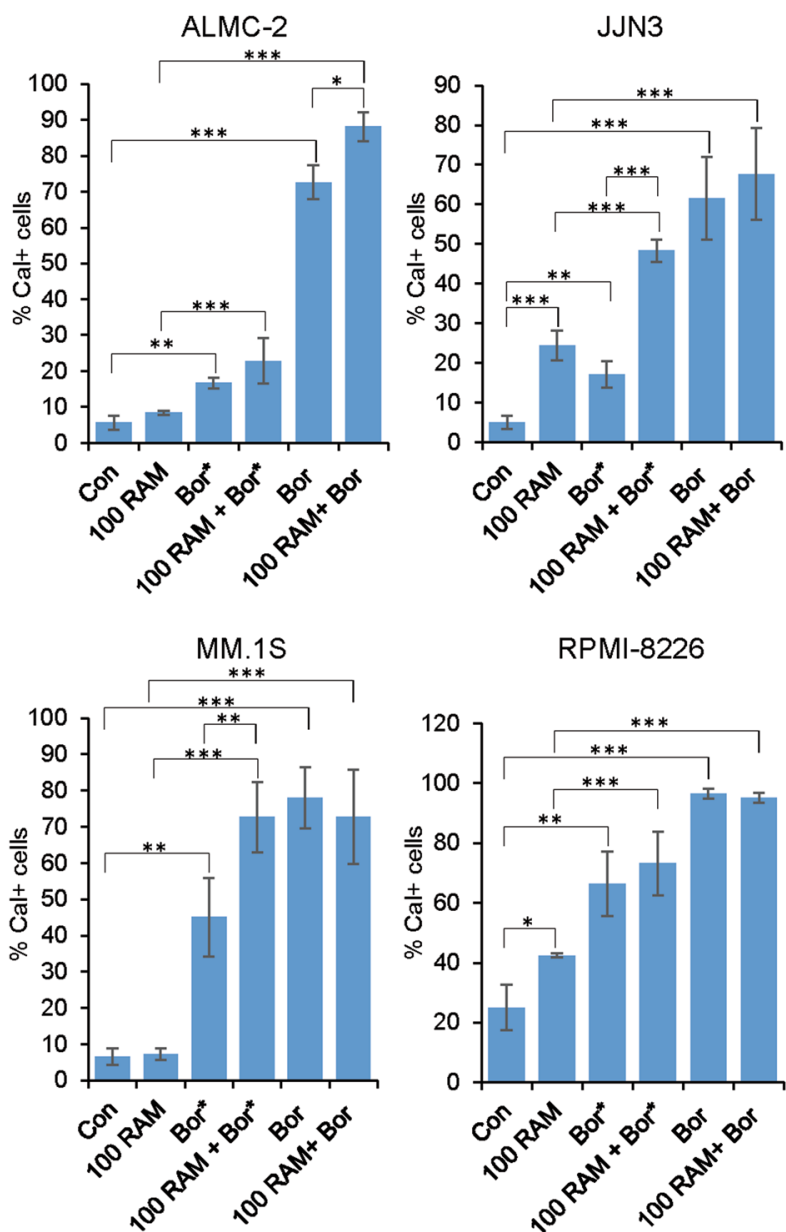

Fig. 4 Combination treatment with RAM2061 and PI leads to induction of the ICD pathway markers. MM cells were incubated with RAM2061 (50 or $100 \mathrm{nM}$ ) for $48 \mathrm{~h}$ with or without sequential (added after $24 \mathrm{~h}$, denoted by*) or concurrent Pl treatment (Bor: $3 \mathrm{nM}$ (MM.1S), 5 nM (JJN3 and RPMI-8226), 15 nM (ALMC-2), Carf: 20 nM). a Immunoblot analysis of secreted HMGB1 was performed using media collected from cells. b, c Cell surface levels of calreticulin were measured by flow cytometry. Data are expressed as the average percentage of calreticulin positive $(n=3$, data are displayed as mean \pm stdev, ${ }^{*}$ denotes $p<0.05 .{ }^{* *}$ denotes $p<0.01$. ${ }^{* *}$ denotes $p<0.001$ per t-test). Blots are representative of three independent experiments 
a

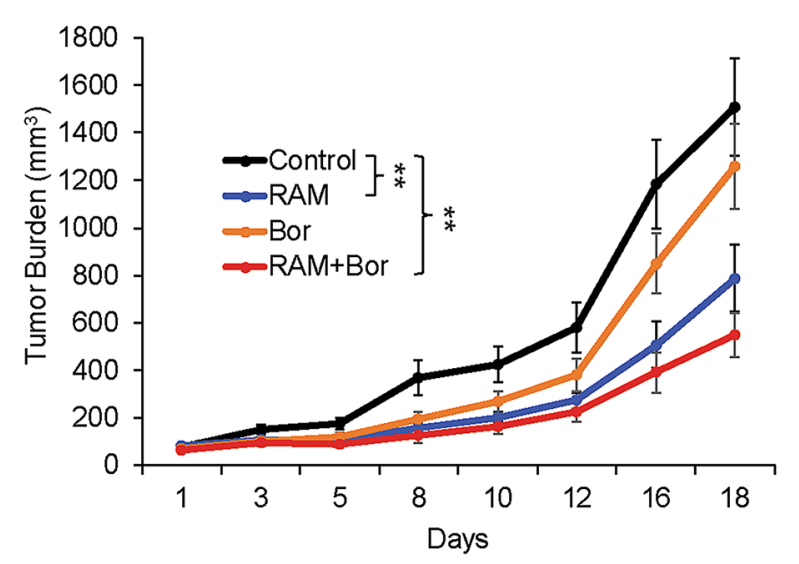

c

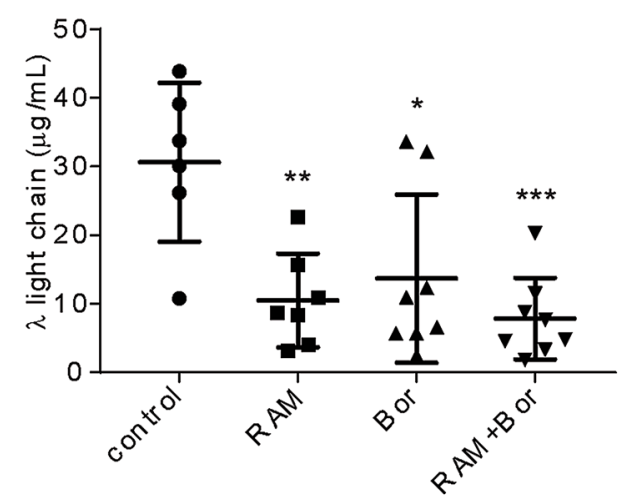

b

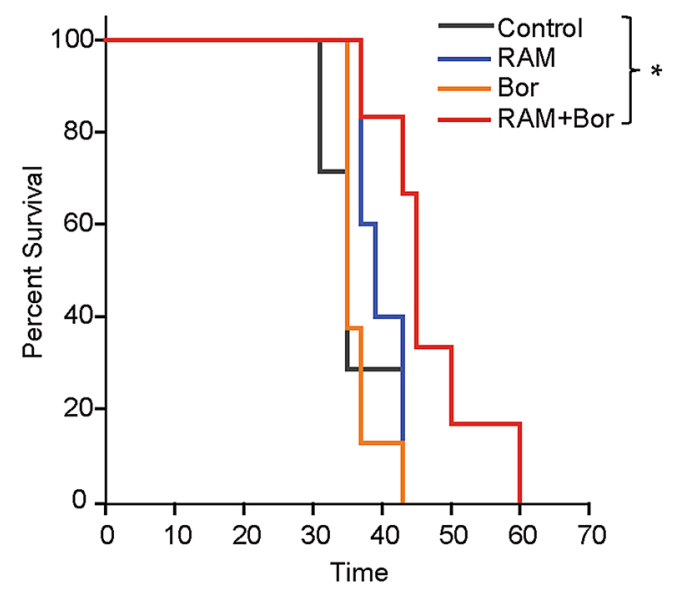

Fig. 5 Effects of RAM2061 and/or bortezomib treatment in MM mouse xenografts. a Tumor growth curves for MM.1S flank xenografts. ( $n=8$ mice per group, data are displayed as mean \pm SEM, denotes $p<0.01$ per mixed-linear mixed model). b Kaplan-Meier curve for MM.1S flank xenografts showing time to sacrifice. Mice were treated twice-weekly with either solvent only control (PBS), RAM2061, Bort, or a combination of RAM2061 and Bort. Mice were euthanized when tumors reached $2000 \mathrm{~mm}^{3}$. ${ }^{*}$ denotes $p<0.05$ per long rank test. c Levels of human $\lambda$ light chain blood samples drawn on day 9 post-initiation of treatment. ${ }^{*}$ denotes $p<0.05,{ }^{* *} p<0.01$ and ${ }^{* * *} p<0.001$ per t-test

IRE1, and p-eIF2a. Our current and previous studies have demonstrated that GGSIs induce all three arms of the UPR [23-25]. While our current studies showed several UPR markers that were further upregulated following combination therapy, the underlying mechanism does not appear to be a direct consequence of accumulation of intracellular MP as the addition of PI did not enhance the effects of the GGSI (Additional file 1: Fig. S8). Prior work has demonstrated that PIs can induce utilization of non-proteasome-mediated degradation pathways such as aggresomes and autophagosomes, thus alleviating the ER stress [31-34]. However, our prior studies with IBP inhibitors demonstrated that these agents do not increase aggresome numbers in MM cells and that that the accumulated intracellular MP induced by these inhibitors is not a substrate for aggresomes, autophagosomes or lysosomes [35]. Notably, the effect of PIs on augmenting RAM2061-induced upregulation of UPR markers was both concentration- and time-dependent. Concurrent exposure results in less induction of the UPR pathway, likely as a result of the more rapid effects of bortezomib on this pathway relative to RAM2061, with subsequent decrease in markers as cells undergo apoptosis. These results likely contribute to the effects observed in the MTT assays, where concurrent treatment appeared to induce less robust effects on cytotoxicity (as indicated by Cl's showing additive/antagonistic interactions) than sequential treatment strategies. Further studies are needed to dissect the mechanisms underlying the interactions between the two classes of drugs on the UPR and apoptotic pathways and to explore alternative dosing schedules that would maximize in vivo efficacy.

There is rationale in the literature to suggest that use of IBP pathway inhibitors would be of therapeutic value as anti-cancer agents. Two studies using the GGDPS inhibitor GGOHBP showed in vivo reduction in tumor burden 
in both adrenal and prostate cancer xenografts [36, 37]. Another preclinical study using a series of thienopyrimidine-based GGDPS inhibitors and the a Vk"MYC transgenic mouse model demonstrated that GGDPS inhibition decreased serum MP levels [38]. Our own studies with RAM2061 or a similar structural analog (VSW1198), show single agent activity in both MM.1S flank xenografts as well as pancreatic cancer xenografts [24, 39]. To date, there is one account in the literature that evaluates the benefit of combining IBP pathway inhibitors with PI therapy. This study shows that statins, which inhibit HMG-CoA reductase in the mevalonate pathway and lead to downstream disruption in isoprenoid synthesis, enhanced induction of apoptosis and UPR markers when used in combination with bortezomib in $t(4 ; 14)$-positive MM cells [40]. Furthermore, using add-back studies they demonstrate that GGPP depletion drives apoptosis and UPR activation in their model. Our present studies have demonstrated synergy between the GGSI and bortezomib in $\mathrm{t}(4 ; 14)$-negative MM cell lines that have other high-risk cytogenetic abnormalities including $t(14 ; 16)$ or $\operatorname{del}(17 \mathrm{p})[26,41]$.

While PIs remain the backbone of MM treatment, development of relapsed/refractory disease has led many researchers to begin testing combination PI treatment with inhibitors of lesser explored pathways. Okabe et al. showed that combined treatment with carfilzomib and CUDC-907, a dual PI3-K and histone deacetylase inhibitor, enhanced cellular toxicity in human MM cell lines when compared to single-drug treatment [42]. In another report, inhibition of phosphoglycerate dehydrogenase (PHGDH) using the inhibitor NT-503, significantly potentiated the cytotoxic effect of bortezomib in seven out of eight tested MM cell lines [43]. Furthermore, combination treatment with NCT-503 and bortezomib displayed enhanced anti-tumor effects in the 5T33MM mouse model when compared to single drug treatments. Together, these studies along with our own demonstrate the therapeutic potential of targeting novel pathways in conjunction with the mainstay PIs and may one day lead to the development of new treatment combinations.

Here we demonstrate that in addition to UPR activation, combination GGSI and PI enhanced the activation of the ICD pathway. While it has been overlooked in favor of studying direct cytotoxic effects, understanding and harnessing ICD is now considered equally important in evaluating a drugs mechanism of action $[44,45]$. The ICD pathway involves the release of damage-associated molecular patterns (DAMPs) by the cancer cell, which attract and activate antigen-presenting cells leading to antitumor immunity [46]. In culture, bortezomib has been shown to be capable of inducing ICD and antitumor immunity by initiating the uptake of Hsp90-expressing human MM cells by dendritic cells [47]. In our studies, bortezomib was the most potent inducer of ICD markers (calreticulin and HMGB1) as a single agent. There are no accounts in the literature describing either carfilzomib or GGSI effects in inducing ICD, however we demonstrate that both compounds can induce HMGB1 release and calreticulin translocation in a cell-line dependent manner. Future experiments dissecting the role ICD induction by PI/GGSI combination therapies plays in vivo are needed.

\section{Conclusion}

In conclusion, our cell culture studies reveal that combination treatment with GGSI and PIs (bortezomib or carfilzomib) increases MM cell death and UPR activation and that this effect is dependent on the timing of drug exposure. Furthermore, we demonstrate that PI/GGSI treatment can potentiate upregulation of markers associated with the immunogenic cell death pathway. These studies are the first to demonstrate the feasibility of combining GGSI and PI therapy in vivo and show evidence of increased efficacy, paving the way towards future preclinical and clinical studies evaluating this novel strategy of disrupting protein homeostasis via multiple mechanisms in MM.

\section{Supplementary Information}

The online version contains supplementary material available at https://doi. org/10.1186/s40164-022-00261-6.

Additional file 1. Additional figures and Tables.

\section{Acknowledgements}

Not applicable.

\section{Authors' contributions}

SLH and SAH were responsible for the conception and design of the study, as well as writing the manuscript. SLH, MLV, JTW and SAH all performed experiments and analyzed data. LMS conducted statistical analysis. GT performed pathology review. All authors read and approved the final manuscript.

\section{Funding}

This work was supported by the National Institutes of Health (Grants P30 CA036727 and 1R01CA258621), the American Society of Hematology and the State of Nebraska Department of Health and Human Services (2020-23).

Availability of data and materials

All data generated or analyzed during this study are included in this published article (and its Additional files). The datasets analyzed during this study are available from the corresponding author on reasonable request.

\section{Declarations}

Ethics approval and consent to participate Not applicable.

Consent for publication

Not applicable. 


\section{Competing interests}

SAH has received honoraria from BMS/Celgene, Genentech, GSK, Janssen, Oncopeptides, Secura Bio, Takeda. The authors declare that they have no competing interests.

\section{Author details}

'Division of Oncology and Hematology, Department of Internal Medicine, University of Nebraska Medical Center, Omaha, NE 68198, USA. ${ }^{2}$ Department of Biostatistics, University of Nebraska Medical Center, Omaha, NE, USA. ${ }^{3}$ Department of Pathology and Microbiology, University of Nebraska Medical Center, Omaha, NE, USA.

Received: 12 November 2021 Accepted: 25 January 2022

Published online: 09 February 2022

\section{References}

1. Obeng EA, Carlson LM, Gutman DM, Harrington WJ Jr, Lee KP, Boise LH. Proteasome inhibitors induce a terminal unfolded protein response in multiple myeloma cells. Blood. 2006;107:4907-16.

2. Utley A, Lipchick B, Lee KP, Nikiforov MA. Targeting multiple myeloma through the biology of long-lived plasma cells. Cancers. 2020;12:2117.

3. Cenci S, Sitia R. Managing and exploiting stress in the antibody factory. FEBS Lett. 2007;581:3652-7.

4. Kubicki T, Gil L, Dytfeld D. Endoplasmic reticulum stress and proteasome inhibitors in multiple myeloma: room for improvement. Pol Arch Intern Med. 2021;131:361-8.

5. Wirth M, Schick M, Keller U, Kronke J. Ubiquitination and ubiquitin-like modifications in multiple myeloma: biology and therapy. Cancers. 2020;12:3764

6. Ri M. Endoplasmic-reticulum stress pathway-associated mechanisms of action of proteasome inhibitors in multiple myeloma. Int J Hematol. 2016;104:273-80.

7. Guerrero-Garcia TA, Gandolfi S, Laubach JP, Hideshima T, Chauhan D, Mitsiades $C$, et al. The power of proteasome inhibition in multiple myeloma. Expert Rev Proteomics. 2018;15:1033-52.

8. Gandolfi S, Laubach JP, Hideshima T, Chauhan D, Anderson KC, Richardson PG. The proteasome and proteasome inhibitors in multiple myeloma. Cancer Metastasis Rev. 2017;36:561-84.

9. Moreau P, Kumar SK, San Miguel J, Davies F, Zamagni E, Bahlis N, et al. Treatment of relapsed and refractory multiple myeloma: recommendations from the International Myeloma Working Group. Lancet Oncol. 2021;22:e105-18.

10. Gandhi UH, Cornell RF, Lakshman A, Gahvari ZJ, McGehee E, Jagosky MH, et al. Outcomes of patients with multiple myeloma refractory to CD38targeted monoclonal antibody therapy. Leukemia. 2019;33:2266-75.

11. Dhanasiri S, Hollier-Hann G, Stothard C, Dhanda DS, Davies FE, RodriguezOtero P. Treatment patterns and outcomes in triple-class exposed patients with relapsed and refractory multiple myeloma: findings from the multinational ITEMISE Study. Clin Ther. 2021;43:1983-96.

12. Haney SL, Wills VS, Wiemer DF, Holstein SA. Recent advances in the development of mammalian geranylgeranyl diphosphate synthase inhibitors. Molecules. 2017;22:886.

13. Zhou X, Ferree SD, Wills VS, Born EJ, Tong H, Wiemer DF, et al. Geranyl and neryl triazole bisphosphonates as inhibitors of geranylgeranyl diphosphate synthase. Bioorg Med Chem. 2014;22:2791-8.

14. Wills VS, Allen C, Holstein SA, Wiemer DF. Potent triazole bisphosphonate inhibitor of geranylgeranyl diphosphate synthase. ACS Med Chem Lett. 2015;6:1195-8

15. Foust BJ, Allen C, Holstein SA, Wiemer DF. A new motif for inhibitors of geranylgeranyl diphosphate synthase. Bioorg Med Chem. 2016;24:3734-41.

16. Allen C, Kortagere S, Tong H, Matthiesen RA, Metzger Jl, Wiemer DF, et al. Olefin isomers of a triazole bisphosphonate synergistically inhibit geranylgeranyl diphosphate synthase. Mol Pharmacol. 2017;91:229-36.

17. Wills VS, Metzger Il, Allen C, Varney ML, Wiemer DF, Holstein SA. Bishomoisoprenoid triazole bisphosphonates as inhibitors of geranylgeranyl diphosphate synthase. Bioorg Med Chem. 2017;25:2437-44.

18. Matthiesen RA, Varney ML, Xu PC, Rier AS, Wiemer DF, Holstein SA. alpha-Methylation enhances the potency of isoprenoid triazole bisphosphonates as geranylgeranyl diphosphate synthase inhibitors. Bioorg Med Chem. 2018;26:376-85.

19. Bhuiyan NH, Varney ML, Bhattacharya DS, Payne WM, Mohs AM, Holstein SA, et al. omega-Hydroxy isoprenoid bisphosphonates as linkable GGDPS inhibitors. Bioorg Med Chem Lett. 2019;29:126633.

20. Goetz DB, Varney ML, Wiemer DF, Holstein SA. Amides as bioisosteres of triazole-based geranylgeranyl diphosphate synthase inhibitors. Bioorg Med Chem. 2020;28:115604.

21. Fairweather AER, Goetz DB, Schroeder CM, Bhuiyan NH, Varney ML, Wiemer DF, et al. Impact of alpha-modifications on the activity of triazole bisphosphonates as geranylgeranyl diphosphate synthase inhibitors. Bioorg Med Chem. 2021;44:116307.

22. Gomes AQ, Ali BR, Ramalho JS, Godfrey RF, Barral DC, Hume AN, et al. Membrane targeting of Rab GTPases is influenced by the prenylation motif. Mol Biol Cell. 2003;14:1882-99.

23. Holstein SA, Hohl RJ. Isoprenoid biosynthetic pathway inhibition disrupts monoclonal protein secretion and induces the unfolded protein response pathway in multiple myeloma cells. Leuk Res. 2011;35:551-9.

24. Haney SL, Chhonker YS, Varney ML, Talmon G, Smith LM, Murry DJ, et al. In vivo evaluation of isoprenoid triazole bisphosphonate inhibitors of geranylgeranyl diphosphate synthase: impact of olefin stereochemistry on toxicity and biodistribution. J Pharmacol Exp Ther. 2019;371:327-38.

25. Born EJ, Hartman SV, Holstein SA. Targeting HSP90 and monoclonal protein trafficking modulates the unfolded protein response, chaperone regulation and apoptosis in myeloma cells. Blood Cancer J. 2013;3:e167.

26. Arendt BK, Ramirez-Alvarado M, Sikkink LA, Keats JJ, Ahmann GJ, Dispenzieri A, et al. Biologic and genetic characterization of the novel amyloidogenic lambda light chain-secreting human cell lines, ALMC-1 and ALMC-2. Blood. 2008;112:1931-41.

27. Holstein SA, Hohl RJ. Interaction of cytosine arabinoside and lovastatin in human leukemia cells. Leuk Res. 2001;25:651-60.

28. Okazuka K, Ishida T. Proteasome inhibitors for multiple myeloma. Jpn J Clin Oncol. 2018;48:785-93.

29. Kraus M, Bader J, Geurink PP, Weyburne ES, Mirabella AC, Silzle T, et al. The novel $\beta 2$-selective proteasome inhibitor LU-102 synergizes with bortezomib and carfilzomib to overcome proteasome inhibitor resistance of myeloma cells. Haematologica. 2015;100:1350-60.

30. Yoshida T, Ri M, Kanamori T, Aoki S, Ashour R, Kinoshita S, et al. Potent anti-tumor activity of a syringolin analog in multiple myeloma: a dual inhibitor of proteasome activity targeting $\beta 2$ and $\beta 5$ subunits. Oncotarget. 2018;9:9975-91.

31. Hoang B, Benavides A, Shi Y, Frost P, Lichtenstein A. Effect of autophagy on multiple myeloma cell viability. Mol Cancer Ther. 2009;8:1974-84.

32. Riz I, Hawley TS, Hawley RG. KLF4-SQSTM1/p62-associated prosurvival autophagy contributes to carfilzomib resistance in multiple myeloma models. Oncotarget. 2015;6:14814-31.

33. Abdel Malek MA, Jagannathan S, Malek E, Sayed DM, Elgammal SA, Abd El-Azeem HG, et al. Molecular chaperone GRP78 enhances aggresome delivery to autophagosomes to promote drug resistance in multiple myeloma. Oncotarget. 2015;6:3098-110.

34. Hideshima T, Bradner JE, Wong J, Chauhan D, Richardson P, Schreiber $\mathrm{SL}$, et al. Small-molecule inhibition of proteasome and aggresome function induces synergistic antitumor activity in multiple myeloma. Proc Natl Acad Sci USA. 2005;102:8567-72.

35. Dykstra KM, Allen C, Born EJ, Tong H, Holstein SA. Mechanisms for autophagy modulation by isoprenoid biosynthetic pathway inhibitors in multiple myeloma cells. Oncotarget. 2015;6:41535-49.

36. Reilly JE, Neighbors JD, Hohl RJ. Targeting protein geranylgeranylation slows tumor development in a murine model of prostate cancer metastasis. Cancer Biol Ther. 2017;18:872-82.

37. Reilly JE, Neighbors JD, Tong H, Henry MD, Hohl RJ. Targeting geranylgeranylation reduces adrenal gland tumor burden in a murine model of prostate cancer metastasis. Clin Exp Metastasis. 2015;32:555-66.

38. Lacbay CM, Waller DD, Park J, Gomez Palou M, Vincent F, Huang XF, et al. Unraveling the prenylation-cancer paradox in multiple myeloma with novel geranylgeranyl pyrophosphate synthase (GGPPS) inhibitors. J Med Chem. 2018;61:6904-17. 
39. Haney SL, Varney ML, Chhonker Y, Talmon G, Smith LM, Murry DJ, et al. In vivo evaluation of combination therapy targeting the isoprenoid biosynthetic pathway. Pharmacol Res. 2021;167:105528.

40. Longo J, Smirnov P, Li Z, Branchard E, van Leeuwen JE, Licht JD, et al. The mevalonate pathway is an actionable vulnerability of $\mathrm{t}(4 ; 14)$-positive multiple myeloma. Leukemia. 2021;35:796-808.

41. Greenstein S, Krett NL, Kurosawa Y, Ma C, Chauhan D, Hideshima T, et al. Characterization of the MM.1 human multiple myeloma (MM) cell lines: a model system to elucidate the characteristics, behavior, and signaling of steroid-sensitive and -resistant MM cells. Exp Hematol. 2003;31:271-82.

42. Okabe S, Tanaka Y, Gotoh A. Targeting phosphoinositide 3-kinases and histone deacetylases in multiple myeloma. Exp Hematol Oncol. 2021;10:19.

43. Elsaadi S, Steiro I, Abdollahi P, Vandsemb EN, Yang R, Slordahl TS, et al. Targeting phosphoglycerate dehydrogenase in multiple myeloma. Exp Hematol Oncol. 2021;10:3.

44. Serrano-Del Valle A, Anel A, Naval J, Marzo I. Immunogenic cell death and immunotherapy of multiple myeloma. Front Cell Dev Biol. 2019;7:50.

45. Fabian KP, Wolfson B, Hodge JW. From immunogenic cell death to immunogenic modulation: select chemotherapy regimens induce a spectrum of immune-enhancing activities in the tumor microenvironment. Front Oncol. 2021;11:728018.

46. Wang YJ, Fletcher R, Yu J, Zhang L. Immunogenic effects of chemotherapy-induced tumor cell death. Genes Dis. 2018;5:194-203.

47. Spisek R, Charalambous A, Mazumder A, Vesole DH, Jagannath S, Dhodapkar MV. Bortezomib enhances dendritic cell (DC)-mediated induction of immunity to human myeloma via exposure of cell surface heat shock protein 90 on dying tumor cells: therapeutic implications. Blood. 2007;109:4839-45.

\section{Publisher's Note}

Springer Nature remains neutral with regard to jurisdictional claims in published maps and institutional affiliations.

- fast, convenient online submission

- thorough peer review by experienced researchers in your field

- rapid publication on acceptance

- support for research data, including large and complex data types

- gold Open Access which fosters wider collaboration and increased citations

- maximum visibility for your research: over 100M website views per year

At BMC, research is always in progress.

Learn more biomedcentral.com/submissions 